\title{
Inclusivity and Dementia: Health Services Planning with Individuals with Dementia
}

\section{Inclusivité et démence : planification des services de santé pour les personnes atteintes de démence}

Effective inclusion requires action at multiple levels by individuals with dementia, care partners, service providers and funding organizations.

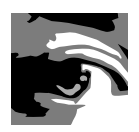

by KYLE WHITFIELD, MSC, PHD

Assistant Professor, Department of Extension

University of Alberta, Edmonton, $A B$

SUSAN WISMER, MED, PHD

Chair, Associate Professor, Department of Environment and Resource Studies University of Waterloo, Waterloo, ON

\begin{abstract}
Historically, Alzheimer societies have identified the care partners of persons with dementia as their central clients. This focus is broadening to include the person with the disease, as well. This paper presents the results of a Canadian research study addressing organizational considerations related to effective inclusion of persons with dementia in planning and decision-making about health services and programs. Our findings suggest that effective inclusion requires action at multiple levels by individuals with dementia, care partners and friends; service organizations and providers; and funding organizations. Additional research is needed to explore the applicability of these findings to other organizations in different localities and to examine emergent
\end{abstract}


themes further. Of these, one that has received little attention to date concerns the potential risks associated with effective inclusion.

\section{Résumé}

Les sociétés Alzheimer ont toujours considéré les partenaires participant aux soins des personnes atteintes de démence comme étant leurs principaux clients. Cette définition est en train de sélargir pour englober les personnes atteintes de la maladie elles-mêmes. Cet article traite des résultats d'une étude canadienne qui visait à cerner les considérations d'ordre organisationnel liées à l'inclusion efficace des personnes souffrant de démence dans la planification et la prise de décisions relatives aux programmes et aux services de santé. Les conclusions suggèrent que l'inclusion efficace exige la prise de mesures, à des paliers multiples, par les personnes atteintes de démence, les partenaires en matière de soins et les amis, les organismes et les fournisseurs de services, ainsi que les bailleurs de fonds. Des travaux de recherche plus poussés sont nécessaires afin d'explorer la pertinence de ces résultats pour d'autres organismes situés à différents endroits et d'examiner plus en profondeur les thèmes émergents. Le risque, par exemple, est un des éléments auxquels on náa accordé que peu d'attention jusqu' ici.

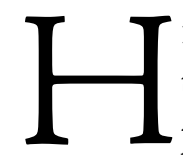

Ealth planning practices are shifting as the Canadian population ages. Those aged 65 and older will constitute $22 \%$ of the Canadian population by the year 2031 (Health Canada 2002). At present, 8\% of all Canadians 65 or older are identified as suffering from dementia, $5.1 \%$ as having Alzheimer disease. If prevalence estimates are accurate, the number of Canadians with dementia will rise to 592,000 by 2021 (Canadian Study of Health and Aging Working Group 1994).

Alzheimer societies are community-based organizations supporting people with dementia and their care partners (Robson and Locke 2002; Eayrs 2002). In Ontario, there are 39 local Alzheimer societies providing information, education, individual and group support, advocacy and referral (Alzheimer Society of Canada 2005). Historically, Alzheimer societies have identified the care partners of persons with dementia as their central client. These organizations now want stronger direct working relationships with individuals with dementia.

This paper reports on a study examining organizational issues relevant to effective direct inclusion of persons with dementia. The study's purpose has been to identify key considerations for the inclusion in decision-making of community members living with dementia. 


\section{Literature Review}

Literature suggests that one crucial component of good governance systems is that they effectively represent the interests of their stakeholders (Light 2002; Institute on Governance 2005). Good governance is also described as drawing on democracy, social responsibility and the public good as guiding principles for the development of policies and for setting goals regarding citizen participation in organizational decisionmaking (Carver 1997). In this same vein, good governance in the field of healthcare requires organizations to ask how they have helped improve the situation for those who are least well off and how they may avoid excluding others who are typically marginalized with respect to resources and supports (Labonte 2004). Wheatley (2005) and Carver (1997) suggest that participatory leadership approaches are characteristic of good governing systems.

Our review of the literature on inclusion and good governance identified three key areas specifically relevant to organizations serving clients with Alzheimer disease: communication, collaborative management strategies and consumer participation in organizational decision-making.

Communication with people with dementia

Individuals with dementia are rarely consulted regarding their opinions and preferences (Nolan et al. 2002). Wilkinson (2002) refers to persons with dementia as one of the most excluded groups in society.

One recent focus in the dementia care literature is on better understanding the

Some strong feelings about exclusion were perspective of persons with expressed. People talked about "meeting walls," feeling unwelcome and being told that opportunities for involvement and services such as support groups could not be made available. the condition. Recent studies have explored the everyday life experience of people with dementia (Gwyther 1997; Phinney 1998; Snyder 2001; Werezak and Stewart 2002) and questions of personhood and identity (Goldsmith 1996; Kitwood 1997; Killick and Allan 2001). In addition, numerous effective communication techniques have been identified for people with dementia at various stages of the disease process (Carnaby 1997; Stalker et al. 1999; Allan 2001; Smith 2002; Moyes 2002). Results of this research suggest that, at most stages of the illness and to varying degrees, people with dementia can express issues and concerns. Appropriate strategies and flexibility regarding time frames and approaches are important (Allan 2000). 
Much of the research focuses on individuals. (See, for example, Bartlett and Martin 2002; Downs 2000.) Our literature review found little examination of the role that organizations can play as facilitators for including people with dementia in making decisions, planning and evaluating health services in the community. One exception is the Alzheimer's Association of Australia, which has reported on the involvement of people with dementia in Alzheimer organizations and how that involvement might be strengthened through capacity building and resource re-allocation (Eayrs 2002).

\section{Collaborative management strategies}

Community-based, non-profit organizations generally endorse inclusion (Dreessen 2001; Shookner 2002; Ochocka et al. 2002). Prior et al. (1995) describe key organizational characteristics: accountable service providers and decision-makers; involved consumers; accessible information; accessible services; client-directed needs assessments; and identified systems for responding to consumer complaints. Similarly, Chaskin et al. (2001: 91) discuss the need for organizations to engage in ongoing monitoring, consumer feedback and consumer involvement in services, planning and evaluation:

... the more an organization can develop relationships that are authentic rather than token, mutual rather than one-sided, and flexible rather than rigid, the more an organization is likely to be able to connect effectively to its constituency and through this connection, contribute to community capacity.

Kretzmann and McKnight (1993) advocate networking with other similar-minded organizations. Petit (2000) proposes that organizations engaging individuals in decision-making share responsibility with other organizations. Light (2002) identifies one characteristic of high-performing, inclusive, non-profit organizations as capacity to collaborate with other organizations.

Heller et al. (1998) assert that participation is an organization-wide effort, undertaken by stakeholders, staff, management and boards, and that it must be supported with resources and policies. Light (2002) highlights the need for leadership to foster open communication.

Effective inclusion of people with dementia requires that organizations value openness, trust and support; draw out the capabilities of individuals with dementia; and have highly developed sensitivity, listening and counselling skills (Barnett 2000). A "genuine appreciation of their awareness and experience by each and every person in the organization involved with that service" is required (Barnett 2000: 204). Kitwood (1997) states that inclusive organizations are caring, that they work at building trust and fostering cooperation and that they seek to minimize the power differences between staff and clients. 


\section{Consumer participation in organizational decision-making}

Barnes (1997) suggests that service users become more knowledgeable and experience greater confidence when they are involved in planning processes. Expectations of consumers have increased - they want more direct decision-making power (Locke et al. 2003). Public frustration with historically low levels of transparency and public accountability in the healthcare system have also fuelled consumer demand for involvement (Abelson et al. 2004).

Frankish et al. (2002) raise questions about whether there is a positive relationship between participation and organizational effectiveness. In contrast, Jewkes and Murcott (1998) report that community participation results in more cost-effective decisions, contributes to a sense of community control and provides an opportunity to release untapped resources. Citizen participation in decision-making processes can enable citizens to be "freed" from professionals and may allow needs to be assessed more accurately (Rifkin 1996).

\section{Methods}

The methodological approach for this research has been qualitative, using interview techniques derived from appreciative inquiry (Watkins 2001), additional interview techniques, analysis of documents and participant observation techniques from ethnographic research (Emerson et al. 1995), and analytical approaches based in grounded theory (Strauss and Corbin 1998). Such a mixed methods approach is recommended particularly for exploratory research as one means of addressing the need for data richness and for purposes of triangulation (Neuman 2003; Palys 2003).

We conducted 37 in-depth, semi-structured individual interviews, with three groups of informants: paid and volunteer staff, people with dementia and identified "experts." The literature review, which we carried out prior to the interviews, provided us with a framework for our selection of groups of interviewees and for the development of questions. Our decision to carry out a relatively small number of in-depth exploratory interviews with a mixed group of key informants was based on our desire to generate a rich base of foundation findings that could provide the basis for future investigation. We also carried out document reviews and engaged in participant observation, focusing on the experiences of the two Ontario Alzheimer organizations that served as case studies for our research. Case study organizations were purposively selected; both directors requested involvement in our research because their organizations were interested in moving towards greater consumer inclusion.

Twelve interviewees were selected purposively, in consultation with executive directors from the two case study organizations, to include key actors in each organization: staff members, volunteers and board members. Interviews explored current and potential involvement of persons with dementia and identified barriers to participation. 
Eleven individuals with a diagnosis of Alzheimer disease, who are in the early to middle stages of the disease process, were contacted through references from the two Alzheimer Society case study organizations, through an online request to the chat room associated with the Dementia Advocacy Support Network International (DASNI) and through references from dementia care service providers. These interviews examined present and desired levels of involvement with local Alzheimer organizations.

Identified experts in the field of dementia care $(n=14)$ included clinicians, researchers, support group facilitators and individuals identified as leading advocates. These experts were identified through Alzheimer networks and from the literature. Questions for experts were developed in response to key themes and issues emerging from first-stage analysis of interviews with organizational representatives and people with dementia.

The first author carried out all interviews individually. They were tape-recorded and transcribed. Interviews with organizational representatives and people with dementia were carried out concurrently, followed by interviews with experts. Data analysis was carried out sequentially. Transcribed results of each interview were compared cumulatively within each subgroup and then across subgroups in order to identify general patterns and themes. Key words and phrases were coded and grouped into categories representing major concepts derived from the data. Coded results from the interviews were then analyzed in relation to relevant literature in order to develop a proposed set of key considerations for organizational practice. Next, these considerations were evaluated and further refined in partnership with the two case study organizations and with interviewees with dementia, who were sent a two-page summary. Finally, based on feedback received, analysis of themes and subthemes was further refined.

\section{Summary of Findings}

Our interviews with people with dementia indicated that inclusion is important to them. They want more opportunities to be involved in decision-making. One individual, wishing to be involved in creating community awareness about dementia, said, "I have done less of this awareness work because that has not been made available to me. ... I need the Society to open the door" (PWiD01-P1). People with dementia said in our interviews that they want meaningful involvement: "I want to feel like I am adding something to the meeting" (PWiD08-P1).

People with dementia also told us that an organization that includes them as decision-making partners facilitates leadership roles for them; it "makes inclusion happen" and enables people with dementia to connect with one another:

We [volunteers with dementia] are supposed to go and give nice feelings to 
people who have got the problem. Here I am, I have the problem ... but at my stage I am OK. So I can go and talk to people.... They may feel comfortable with me because I have Alzheimer's disease. (PWiD06-P1)

Direct connections to Alzheimer Society chapters are relatively recent and are evolving:

I have seen some real growth in my Alzheimer Society. ... My initial experience was really bad. ... Now I am on a Steering Committee, have been on a panel talk ... , sent to a conference and written an article for their newsletter. (PWiD02-P1)

People with dementia who are involved in decision-making with Alzheimer societies reported feeling better because of their involvement, feeling nurtured and feeling an improvement in their health:

Maybe I feel better just taking action and taking control and I feel good about it and it enhances my overall performance. Or is it actually having an effect on my brain? Whichever way, I can't lose.... You have to work your brain. (PWiD01-P1)

Some strong feelings about exclusion were expressed. People talked about "meeting walls," feeling unwelcome and being told that opportunities for involvement and services such as support groups could not be made available. Several individuals with dementia told us that they had to be persistent because "they [their local Alzheimer Society] were not accommodating" (PWiD02-P1). While these sentiments indicate that there is still considerable work to be done, they also affirm the importance and relevance of initiatives dedicated to inclusion.

Alzheimer societies involved in our study clearly believe that inclusion matters. However, only since 2001, as part of a major policy initiative by provincial and national organizations, have these societies begun to think about new roles for the person with dementia. The case study organizations currently include people with dementia as support group members, as co-facilitators of support groups, as advisory committee members, as friendly visitors, as speakers at Annual General Meetings, as representatives on speakers' panels and as writers of book reviews or other articles in local Alzheimer Society newsletters. At the time of our study, neither organization had people with dementia serving on their board of directors.

Those representing Alzheimer organizations told us that an inclusive organization creates structures that intentionally foster inclusion. Some interviewees saw inclusion as possible because the culture of Alzheimer organizations is friendly, learning orient- 
ed and sincere, and promotes possibility. Key informants suggested that an inclusive organization should have an identified staff person whose role is to ensure successful inclusion.

The societies reported several challenges. A funder focus on care partners makes it difficult to concentrate on developing a strong working partnership with people with dementia. Case study organizations said the progressive nature of the disease also challenges inclusion: "The farther people move along the disease, they have less insight" (HN-05-P1). Resources generally are limited: "We are so focused on our existence and sustainability; it's hard to be innovative when you are just sustaining yourself financially" (S06-P1).

\section{"You can't always take the experience of the person with dementia as fact,... [therefore] we need to be well educated about the disease process in order to balance what they say (which may not be fact) with the process of the disease"}

Interviews with experts corroborated findings from other interviews and additionally indicated that involving people with dementia in selected issues that are most relevant and of greatest interest to them is the most effective approach. One interviewee suggested, for example, asking people with dementia to act as resource persons to be consulted on relevant issues

by the board of directors, as an alternative to board representation. Other recommendations included evaluating organizational constitutions for inclusivity; updating language, membership and objectives of the organization and its programs; creating new roles that are meaningful for people with dementia; providing skill-building opportunities for staff and for people with dementia; and fostering an organizational culture and a board that is open to change and willing to take risks.

Experts identified risks of participation for people with dementia. Some said that people with dementia who are involved in health service planning processes risk feeling belittled or demeaned if they are misunderstood or if they are frustrated in attempts at communication. Two experts suggested that people with dementia might also be at risk because their lives are already stressful. If organizational involvement creates additional stress, stress levels could become overwhelming.

Experts talked also about organizational risks. Not being able to respond to all the requests of a person with dementia exposes organizations to criticism from those whom they mean to serve. Consumer involvement may shift power from staff, volunteers, board members and caregivers to people with dementia with the result that 
"there is danger that some people may feel their interests are not being as strongly represented as before" (EXPRT15-P1). Another identified risk was that comments made by people with dementia might not always be accurate: "You can't always take the experience of the person with dementia as fact,... [therefore] we need to be well educated about the disease process in order to balance what they say (which may not be fact) with the process of the disease" (EXPRT09-P1). (It is important to note here that people without Alzheimer disease may also hold inaccurate views.)

Experts we interviewed differed about the degree of caution needed regarding inclusion of persons with dementia in organizational initiatives. More cautious experts talked about people with dementia being at risk of exploitation and in need of protection (EXPRT07-P1) and "containment" (EXPRT11-P1). Others, representing a middle ground, talked about weighing the risks and the benefits: "We don't want to swing the pendulum too far because there are unique elements to the disease." Less cautious experts said: "We live with risk every day, and people with dementia cannot be denied the opportunity to speak about their own experience. This is paternalistic" (EXPRT06-P1); "It's a risk to not hear their voice" (EXPRT-11-P1); "Get over it, we have to take a risk, we can't live in a state of fear - life is a risk" (EXPRT09-P1). Experts differed in their views about the degree of consideration needed when involving persons with dementia in health services planning and decision-making. There was consensus, however, that organizational initiatives directed towards inclusion are not risk-free for the person with dementia or for organizations.

\section{Discussion: Towards Inclusive Health Services Delivery for Persons with Dementia}

Our results indicate that major shifts will be required for most organizations if they are to move from a focus on providing "support" or services to a broader focus on including people with dementia as partners in planning and decision-making with regard to programs and services. There is good opportunity for this: our case studies indicated that people with dementia are typically embedded within a broader community context, one that moves beyond relationships with family members, friends and neighbours to include agencies and organizations in supporting communities, offering a variety of opportunities for enhancing inclusion at multiple levels (Figure 1).

We have concluded that organizations that include people with dementia in decision-making must be adaptive and flexible enough to accommodate changing needs. Issues of scale may be critical in increasing adaptive capacities for inclusive organizations serving people with dementia. Although improved communication strategies at the level of the individual are important in maximizing the benefits of service provi- 
FIGURE 1. Connections between individuals with dementia and their broader community

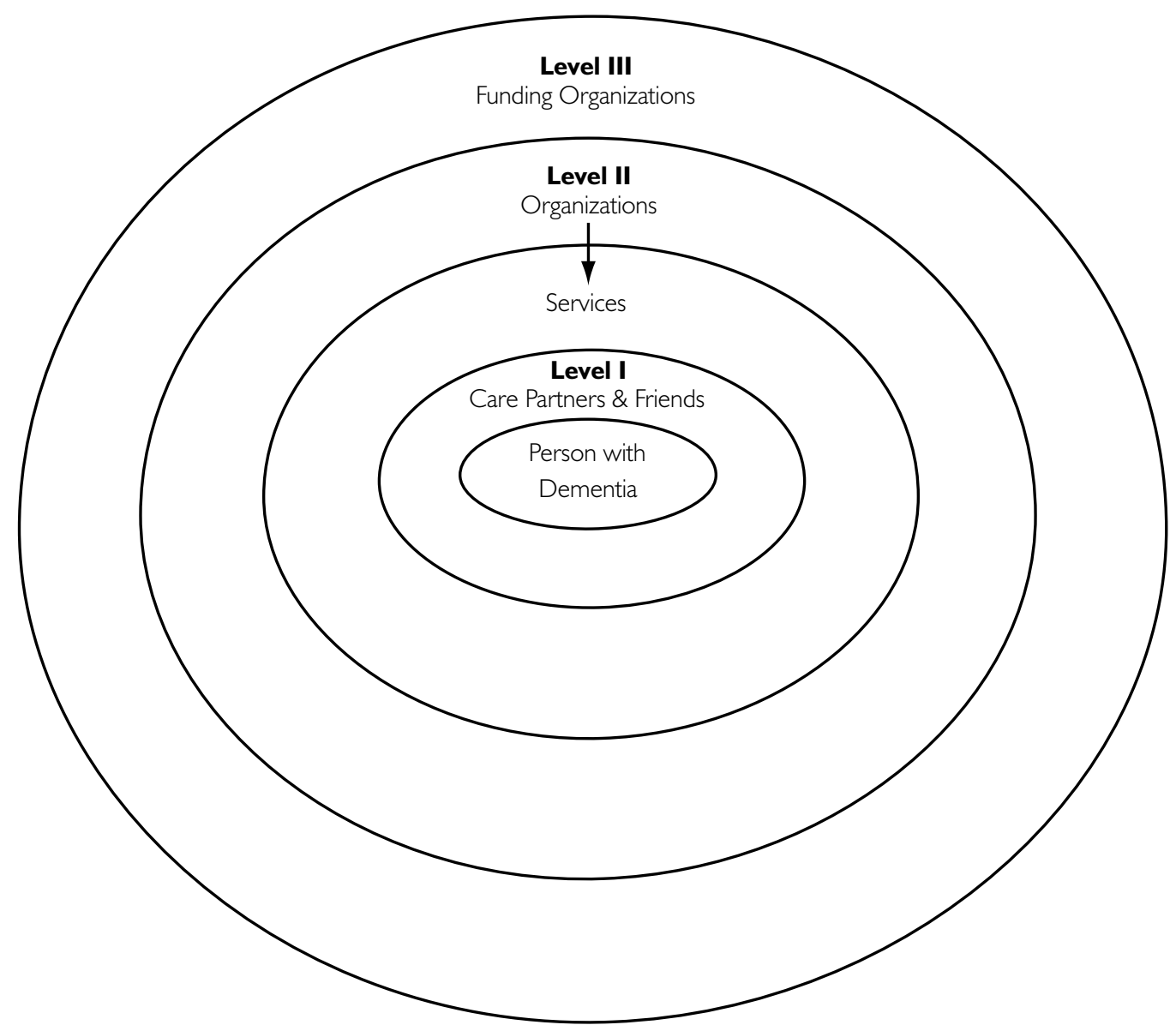

sion for people with dementia, they will not necessarily create organizations that are effectively inclusive. Specific strategies for change at the level of the organization are required and include a vision, structure and board that accommodate people with dementia; leadership opportunities for people with dementia; acknowledgment that inclusion requires resources; and development of an organizational culture that is ready and willing to move towards inclusion. At the meta-level (Level III in Figure 1) of funders and government-based funding agencies, the critical role of resources in supporting organizational change means that changes at the policy and program levels are also necessary. While our study focuses on addressing a research gap with respect to organizations and organizational change (Level II in Figure 1), our findings also suggest that effective inclusive organizations will be nested in comprehensive and ongoing initiatives at all three levels. For example, a funder focus on caregiver support was identified as a major challenge by our organizational interviewees. 
Findings from this study suggest three important areas for further research and analysis: governance, resource requirements and risk.

\section{Organizational governance issues}

Our interviews with individuals with dementia confirmed their interest in participating in decision-making roles as board or committee members in Alzheimer organizations. Organizational representatives also were strongly committed to inclusion. There are, however, limits that need to be considered.

Dementia, and Alzheimer disease in particular, is a changing disease. The ability of any individual to be involved as a board or committee member is likely to be time limited and may vary considerably within that period depending on individual factors (e.g., fatigue, stress, wellness) and environmental considerations (e.g., level and type of organizational supports, communication protocols, etc.). While these people have the ability to attend meetings, grasp the process and reflect on and communicate about relevant board or committee issues, this is likely to be possible mainly in the earlier stages of the disease. The changeable nature of dementia creates challenges for affected individuals and also for other board or committee members. Sensitivity, developed listening skills and a partnership approach to inclusion are necessary. Honest communication about anticipated fluctuations and long-term changes in cognitive capacity is likely to be important at the outset among all involved.

Frankish et al. (2002) suggest that there are mixed indications in the literature about citizens' representation. In their exploration of the role of citizens on regional health boards, the authors found no evidence of better decisions being made or resources being used more efficiently because of citizen representation. Barnes (1997) also points out that citizens involved in a long-term planning process are not a reliably representative sample of the user population simply by virtue of their experience. This broader research on citizen representation indicates that people with dementia, like other identified groups, are not homogeneous, making representation a challenge. Effective inclusion must take into account the need to support people in representing the full range of their constituencies (Pitkin 1967).

\section{Resource-related issues}

Eayrs (2002) reports lack of resources as one of the biggest barriers to inclusion in the Alzheimer's Association of Australia. In Ontario, the 39 Alzheimer societies receive varying proportions of their funding from the provincial government (Société Alzheimer Society Ontario 2005). In general, there is significant reliance upon the Ontario Ministry of Health and Long-Term Care. This is not unusual for not-forprofit organizations. Phillips $(1995: 12)$ argues that "it is simply a myth to think that 
... the voluntary sector ... does - or could - operate entirely independently of government."

Government cutbacks mean that voluntary sector organizations, such as local Alzheimer societies, are experiencing reduced funding just as they are being asked to take on a larger share of responsibility for delivering services. At the same time, the demand for services is increasing. There is, typically, a financial risk associated with change: "Funding cuts hamper the capacity of the voluntary sector to carry out its other roles with respect to innovation and moral leadership" (Torjman 1999: 4).

The non-profit organizational management literature suggests that understanding of the current and potential role of the not-for-profit sector is lacking (Prince and Chappell 1994; Phillips 1995; Dreessen 2001). Several authors suggest that governments need to appreciate that voluntary organizations "weave the fabric of society by engaging citizens, and cultivating trust and collaboration among sectors.... [They] act as the social glue that helps bind together the diverse elements of society into a cohesive whole" (Torjman 1999: 7).

\section{Risk}

A third theme emerging from our findings relates to risk. We found agreement that inclusion entails risk, both for individuals and for organizations. We also found considerable disagreement about what constitutes acceptable risk and how to respond to it. Risk perception and risk assessment have emerged as a potentially important area for further investigation regarding organizational inclusion of people with dementia.

\section{Conclusion}

People with dementia in our study would like to play a stronger role in healthcare decision-making. Our findings suggest that there is an important role for Alzheimer societies in "opening the door" for them to do so. Direct relationships between individuals with dementia and Alzheimer organizations are still relatively recent. Currently, much of the research focuses on improvements in methods of communication with individuals with dementia. While communication is critically important, our findings suggest that improved communication is not sufficient to achieve inclusivity. Inclusive organizations need to place priority on good governance; involvement of consumers in service-related decision-making; leadership that fosters open communication and draws out people's capacities and potential for creative problem-solving; and supportive policies and resources. As Figure 1 suggests, effective inclusion requires action at multiple levels by individuals with dementia, care partners and friends; organizations and service providers; and funding organizations.

Because our study is based on only two case studies and our sample size is not 
large, we cannot claim that our results are broadly generalizable. Additional research is needed to explore the relevance of these findings to other organizations in different localities and to examine further the themes that emerged in our study. Of these, one that has received little attention to date is risk.

\section{ACKNOWLEDGMENTS}

The authors would like to thank reviewers for their comments on earlier versions of this paper. This study was supported in part by the Canadian Institutes of Health Research - Institute on Aging and the Scottish Rites Charitable Foundation of Canada.

Correspondence may be directed to: Kyle Whitfield, Faculty of Extension, University of Alberta, Edmonton, Alberta, T6G 2T4, tel: 780-492-2681, fax: 780-492-6735, email: kw16@ualberta.ca

\section{REFERENCES}

Abelson, J., P.G. Forest, J. Eyles, A. Casebeer and G. Mackean. 2004. “Will It Make a Difference If I Show Up and Share? A Citizen's Perspective on Improving Public Involvement Processes for Health System Decision-Making." Journal of Health Services Research Policy 9: 205-212.

Allan, K. 2000. "Drawing Out Views on Services: A New Staff-Based Approach." Journal of Dementia Care 8: 16-19.

Allan, K. 2001. Communication and Consultation: Exploring Ways for Staff to Consult People with Dementia about Services. Bristol, UK: The Policy Press.

Alzheimer Society of Canada. 2005. Retrieved December 9, 2005. <http://www.alzheimer.ca/ english/index.php>.

Barnes, M. 1997. Care, Communities and Citizens. London, UK: Addison Wesley Longman.

Barnett, E. 2000. Including the Person with Dementia in Designing and Delivering Care: "I Need to be Me." London, UK: Jessica Kingsley Publishers.

Bartlett, H. and W. Martin. 2002. "Ethical Issues in Dementia Care Research." In H. Wilkinson, ed., The Perspectives of People with Dementia: Research Methods and Motivations (pp. 47-61). London, UK: Jessica Kingsley Publishers.

Canadian Study of Health and Aging Working Group. 1994."Canadian Study of Health and Aging: Study Methods and Prevalence of Dementia." Canadian Medical Association Journal 150: 899-913.

Carnaby, S. 1997. “'What Do You Think?': A Qualitative Approach to Evaluating Individual Planning Services." Journal of Intellectual Disability Research 41: 225-231.

Carver, J. 1997. Boards That Make a Difference: A New Design for Leadership in Nonprofit and Public Organizations. San Francisco: Jossey-Bass.

Chaskin, R., P. Brown, S. Venkatesh and A. Vidal. 2001. Building Community Capacity. New York: Aldine de Gruyter. 
Downs, M. 2000. "Dementia in a Socio-Cultural Context: An Idea Whose Time Has Come." Ageing and Society 20: 369-375.

Dreessen, E. 2001. "What We Should Know about the Voluntary Sector But Don't Know." Isuma: Canadian Journal of Policy Research 2: 1-15.

Eayrs, A. 2002. Consumer Focus Project. Higgins, Australia: Alzheimer's Association Australia.

Emerson, R., R. Fretz and L. Shaw. 1995. Writing Ethnographic Fieldnotes. Chicago: University of Chicago Press.

Frankish, J., B. Kwan, C. Larsen, P. Ratner and J. Wharf Higgins. 2002. “Challenges of Community Participation in Health-System Decision-Making." Social Science and Medicine 54(10): 1471-1480.

Goldsmith, M. 1996. Hearing the Voice of People with Dementia: Opportunities and Obstacles. London, UK: Jessica Kingsley Publishers.

Gwyther, L. 1997."The Perspective of the Person with Alzheimer Disease: Which Outcomes Matter in Early to Middle Stages of Dementia?" Alzheimer's Disease and Associated Disorders 11: $18-24$.

Health Canada. 2002. Canada's Aging Population. Ottawa: Minister of Public Works and Government Services Canada.

Heller, F., E. Pusic, G. Strauss and B. Wilpert. 1998. Organizational Participation: Myth and Reality. New York: Oxford University Press.

Institute on Governance. 2004. “Board Governance. Fact Sheet." Retrieved December 9, 2005. <http://www.iog.ca>.

Jewkes, R. and A. Murcott. 1998. “Community Representatives: Representing the 'Community'?” Social Science Medicine 46: 843-858.

Killick, J. and K. Allan. 2001. Communication and the Care of People with Dementia. Buckingham, UK: Open University Press.

Kitwood, T. 1997. "The Experience of Dementia." Aging and Mental Health 1: 13-22.

Kretzman, J. and J. McKnight. 1993. Building Communities from the Inside Out. Evanston, IL:

Center for Urban Affairs and Policy Research, Northwestern University.

Labonte, R. 2004. "Social Inclusion/Exclusion: Dancing the Dialect." Health Promotion International 19: 115-121.

Light, P. 2002. Pathways to Nonprofit Excellence. Washington, DC: Brookings Institution Press.

Locke, M., N. Begum and P. Robson. 2003. "Service Users and Charity Governance." In C.

Cornforth, ed., The Governance of Public and Non-Profit Organizations (pp. 57-73). London, UK: Routledge.

Moyes, M. 2002."The Voice of the User Group." Signposts Review 6: 42-44.

Neuman, W.L. 2003. Social Research Methods (5th ed.). Boston: Pearson Education.

Nolan, M., T. Ryan, P. Enderby and D. Reid. 2002.“Towards a More Inclusive Vision of Dementia Care Practice and Research." Dementia 1: 193-211.

Ochocka, J., R. Janzen and G. Nelson. 2002. "Sharing Power and Knowledge: Professional and Mental Health Consumer/Survivor Researchers Working Together in a Participatory Action 
Research Project." Psychiatric Rehabilitation Journal 25: 379-387.

Palys, T.S. 2003. Research Decisions (3rd ed.). Toronto: Thomas Nelson.

Petit, J. 2000. “Strengthening Local Organizations - 'Where the Rubber Hits the Road.” IDS Bulletin 31: 57-67.

Phillips, S.D. 1995. "Redefining Government Relationships with the Voluntary Sector: On Great Expectations and Sense and Sensibility." Voluntary Sector Roundtable Publications. Retrieved December 9, 2005. <http://www.vsr-trsb.net/publications/phillips-e.html>.

Phinney, A. 1998. "Living with Dementia: From the Patient's Perspective." Journal of Gerontological Nursing 24. 8-15.

Pitkin, H. 1967. The Concept of Representation. Berkeley: University of California Press.

Prince, M. and N. Chappell. 1994. Voluntary Action by Seniors in Canada. Victoria, BC: University of Victoria Centre on Aging.

Prior, D., J. Stewart and K. Walsh. 1995. Citizenship: Rights, Community and Participation. London, UK: Pitman.

Rifkin, S. 1996. "Paradigms Lost: Toward a New Understanding of Community Participation in Health Programmes." Acta Tropica 61: 79-92.

Robson, P. and M. Locke. 2002. Changing Voices: Involving People with Dementia in the Alzheimer's Society. Final Evaluation Report. London, UK: Centre for Institutional Studies.

Shookner, M. 2002. An Inclusion Lens: Workbook for Looking at Social and Economic Exclusion and Inclusion. Halifax: Health Canada.

Smith, J. 2002. "From Rhetoric to Reality." Nursing Older People 14: 10-13.

Snyder, L. 2001."The Lived Experience of Alzheimer's - Understanding the Feelings and Subjective Accounts of Persons with the Disease." Alzheimer's Care Quarterly 2: 8-22.

Société Alzheimer Society Ontario. 2005. Retrieved December 9, 2005. <http://alzheimer ontario.org >.

Stalker, K., J. Gilliard and M. Downs. 1999. “Eliciting User Perspectives on What Works.” International Journal of Geriatric Psychiatry 14: 120-134.

Strauss, A. and J. Corbin. 1998. Basics of Qualitative Research. London, UK: Sage Publications. Torjman, S. 1999. Unravelling the Threads of Democracy. Toronto: Caledon Institute of Social Policy.

Watkins, J.M. 2001. Appreciative Inquiry: Change and the Speed of Imaginations. San Francisco: Jossey-Bass.

Werezak, L. and N. Stewart. 2002. "Learning to Live with Early Dementia." Canadian Journal of Nursing Research 34: 67-85.

Wheatley, M. 2005. Finding Our Way: Leadership for an Uncertain Time. San Francisco: BerrettKoehler Publishers.

Wilkinson, H. 2002. The Perspectives of People with Dementia. London, UK: Jessica Kingsley Publishers. 